\title{
The nucleus of IC 342 as a potential twin of the Galactic center
}

\author{
David S. Meier ${ }^{1,2}$ \\ ${ }^{1}$ New Mexico Institute of Mining \& Technology, 801 Leroy Pl., Socorro, NM 87801, USA \\ email: dmeier@nmt.edu \\ ${ }^{2}$ National Radio Astronomy Observatory, P. O. Box O, Socorro, NM 87801, USA
}

\begin{abstract}
The Galactic center (GC), being the closest nucleus, holds a position of privilege in the study of galaxy centers, but because it is edge-on and hidden behind 30 magnitudes of visual extinction it is often difficult to understand the overall structure of the region. Nearby galactic nuclei potentially provide a guide to understanding the large-scale structure of the GC. High resolution maps of molecular line emission along with radio and optical continuum towards the nucleus of the nearby, face-on spiral IC 342 are discussed. Attention is focused on a comparison of the large-scale morphology, gas chemistry, and star formation between the two nuclei. The case is made that IC 342 is one of the best extragalactic templates for the GC. Both have a star formation rate within a factor of two of each other and an ISM morphology characterized by a $\mathrm{R} \sim 300 \mathrm{pc}$ central molecular zone formed from a pair of arms laced with a collection of dense star forming molecular clouds. IC 342 also exhibits a nuclear cluster and associated circumnuclear disk. Whether the nuclear morphology and chemistry in IC 342 is an extension of the disk bar, a separate nuclear bar, or results from radiative/mechanical feedback remains unsettled.
\end{abstract}

Keywords. galaxies: individual (IC 342), galaxies: nuclei, Galaxy: nucleus, ISM: structure

\section{Introduction}

IC 342, one of the closest face-on spiral galaxies, hosts a nucleus that is gas-rich and actively forming stars. The structure and context of IC 342's nucleus is reviewed in the context of the Galactic center (GC). Table 1 summarizes a sample of parameters for each nucleus. In many respects, IC 342's nucleus appears as a potential twin of the GC.

\section{The overall dynamical picture of IC 342's nucleus}

The large scale dynamics of the GC and its connection to star formation has been a topic of long-term discussion. IC 342's face-on nature and observed similarities makes it an important comparison case. IC 342's nucleus is characterized by a bright central molecular zone (CMZ) with molecular mass and spatial extent comparable to the GC. Its morphology is two extended open molecular arms that terminate on a dense, star forming central ring of radius $\sim 100$ pc (e.g. Meier \& Turner 2001). The central ring is dominated by three massive GMCs containing the bulk of the current star formation (about twice the GC star formation rate). Like the GC, a separate circumnuclear disk resides inside the central ring. It is marginally resolved in $10 \mathrm{pc}$ resolution $\mathrm{CO} J=(2-1)$ data and is centered on a $>10^{7} \mathrm{M}_{\odot}$ young ( $<25 \mathrm{Myr}$ ), nuclear cluster (e.g. Böker et al. 1997, Schinnerer et al. 2008). X-ray emission is confined within the central ring and resolves into a ring-filling diffuse, soft component and an unresolved point source at the nuclear cluster (Mak et al. 2008). The X-ray luminosity can be explained purely by star formation (e.g. O stars, stellar winds, HMXBs, SNRs). No supermassive black hole is seen in IC 342 to a mass limit well below the GC, consistent with its smaller bulge (Table 1). 
Table 1. Overview of nuclear parameters for IC 342 and the Galactic center

\begin{tabular}{|lccc|}
\hline Parameter & IC 342 & Galactic center & Notes \\
\hline Distance & $3.3 \mathrm{Mpc}$ & $0.0080 \mathrm{Mpc}$ & 1 \\
Dynamical Mass $(R<100 \mathrm{pc})$ & $2 \times 10^{8} \mathrm{M} \odot$ & $2 \times 10^{8} \mathrm{M} \odot$ & 2 \\
Radius (CMZ) & $400 \mathrm{pc}$ & $300 \mathrm{pc}$ & 3 \\
Molecular Gas Mass $(R<275 \mathrm{pc})$ & $2 \times 10^{7} \mathrm{M}_{\odot}$ & $3 \times 10^{7} \mathrm{M}_{\odot}$ & 4 \\
Ionizing Photon Rate $\left(N_{L y c}\right)$ & $4 \times 10^{52} \mathrm{~s}^{-1}$ & $2 \times 10^{52} \mathrm{~s}^{-1}$ & 5 \\
X-ray Luminosity $(0.5-10 \mathrm{keV})$ & $2 \times 10^{39} \mathrm{erg} \mathrm{s}^{-1}$ & $>4 \times 10^{39} \mathrm{erg} \mathrm{s}^{-1}$ & 6 \\
Mass (SMBH) & $<1 \times 10^{6} \mathrm{M}_{\odot}$ & $\sim 4.1 \times 10^{6} \mathrm{M}_{\odot}$ & 7 \\
Mass of Largest GMC & $3 \times 10^{6} \mathrm{M}_{\odot}$ & $\sim 3 \times 10^{6} \mathrm{M}_{\odot}$ & 8
\end{tabular}

Notes:

1) Saha, Claver \& Hoessel (2002), Reid et al. (2009); 2) Turner \& Hurt (1992), Genzel \& Townes (1987); 3) e.g. Meier \& Turner (2001), Bania (1977); 4) Meier \& Turner (2001), Dahmen et al. (1998); 5) Turner \& Ho (1983), Mezger \& Paul (1979); 6) Bauer, Brandt \& Lehmer (2003), Kong (2003), Kaneda et al. (1997); 7) Boker, van der Marel \& Vacca (1999), e.g. Ghez et al. (2008). 8) Meier \& Turner (2001), Bally et al. (1988)

While the morphology of the nucleus of IC 342 is clear, its interpretation remains unsettled. Two different scenarios have partial support from data, 1) nuclear bar driven star formation and gas morphology (Böker et al. 1997; Meier \& Turner 2005), or 2) a continuation of the large scale spiral arms, together with the central ring being a cavity blown out by feedback from the nuclear cluster starburst (e.g. Turner \& Hurt 1992; Schinnerer et al. 2008). The morphological similarity with other nuclear bars (e.g. Meier \& Turner 2012), the locations of the active star formation at proposed $x_{1}-x_{2}$ orbit intersections and shocked gas chemistry, traced by $\mathrm{CH}_{3} \mathrm{OH}, \mathrm{SiO}$ and $\mathrm{HNCO}$, peaking at the end of the CMZ (Meier \& Turner 2005) favor the first scenario. But the alignment between the inner arms and the large scale spiral pattern and the morphology of the central ring are better explained by the second picture.

\section{Acknowledgements}

The author acknowledges partial support from the NSF grant AST-1009620.

\section{References}

Bania, T. M. 1977, ApJ 216, 381

Bally, J., Stark, A. A., Wilson, R. W., \& Henkel, C. 1988, ApJ 324, 223

Bauer, F. E., Brandt, W. N., \& Lehmer, B. 2003, AJ 126, 2797

Böker, T., Forster-Schreiber, N. M., \& Genzel, R. 1997, AJ 114, 1883

Böker, T., van der Marel, R. P., \& Vacca, W. D. 1997, AJ 118, 831

Dahmen, G., Hüttemeister, S., Wilson, T. L., \& Mauersberger, R. 1998, A\&\&A 331, 959

Genzel, R. \& Townes, C. H. 1987, ARAA 25, 377

Ghez, A. M., Salim, S., Weinberg, N. N., Lu, J. R., Do, T., Dunn, J. K., Matthews, K., Morris, M. R., Yelda, S., Becklin, E. E., Kremenek, T., Milosavljevic, M., \& Naiman, J. 2008, ApJ 689,1044

Kaneda, H., Makishima, K., Yamauchi, S., Koyama, K., Matsuzaki, K., \& Yamasaki, N. Y. 2008, ApJ 491, 638

Kong, A. K. H. 2008, MNRAS 346, 265

Mak, D. S. Y., Pun, C. S. J. \& Kong, A. K. H. 2008, ApJ 686, 995

Meier, D. S. \& Turner, J. L. 2001, ApJ 551, 687

Meier, D. S. \& Turner, J. L. 2005, ApJ 618, 259

Meier, D. S. \& Turner, J. L. 2012, ApJ 755, 104

Mezger, P. G. \& Paul, T. 1979, The large-scale characteristics of the galaxy, Proc. of the Symposium, \& College Park, Md. (Dordrecht: D. Reidel Publishing Co.), 1979, p. 357

Reid, M. J., Menten, K. M., Zheng, X. W., Brunthaler, A., \& Xu, Y. 2009, ApJ 705, 1548 
Saha, A., Claver, J., \& Hoessel, J. G. 2002, AJ 124, 839

Schinnerer, E., Böker, T., Meier, D. S., \& Calzetti, D. 2008, ApJ Lett. 684, 21

Turner, J. L. \& Ho, P. T. P. 1983, ApJ Lett. 268, 79

Turner, J. L. \& Hurt, R. L. 1992, ApJ 384, 72 